\title{
Augment BeiDou real-time precise point positioning using ECMWF data
}

\author{
Kemin Zhu' ${ }^{1}$ Lei Zhao ${ }^{1}$, Wei Wang ${ }^{1}$, Shoujian Zhang ${ }^{1 *}$, Renli Liu ${ }^{2^{*}}$ and Jinling Wang ${ }^{3}$
}

\begin{abstract}
The Chinese BeiDou Navigation Satellite System has shown potential for precise positioning with a comparable accuracy to that of the Global Positioning System (GPS) at the centimeter level for the horizontal component and the subcentimeter level for the vertical component. However, a longer convergence time limits the performance of BeiDou precise point positioning (PPP) compared to the GPS PPP solutions. In this study, we applied the tropospheric delay information, derived from the European Centre of Medium-Range Weather Forecasts (ECMWF) analysis and prediction data, into the simulated real-time BeiDou-only and BeiDou/GPS PPP to augment the solutions. Observations from stations in Southeast Asia, which are capable of tracking the BeiDou constellation from the International GNSS Service (IGS) Multi-GNSS Experiment and Pilot Project (MGEX) network, are processed with different strategies: the standard PPP and the introduced ECMWF-augmented PPP with analysis and prediction data, respectively. The positioning results demonstrate that the ECMWF-augmented BeiDou-only and BeiDou/GPS PPP methods using prediction data perform as well as those using analysis data. In the case of BeiDou-only PPP scenarios, remarkable advancements of $80.6 \%$ for the convergence time are achieved by two ECMWF-augmented PPP solutions with respect to the standard PPP method. For the positioning accuracy, the two proposed augmented PPP methods attain $6.6 \mathrm{~cm}$ in three-dimensional (3D) accuracy when the standard PPP solution get converged $(10 \mathrm{~cm})$, representing a remarkable improvement of $34 \%$. As for the north/east/up component, improvements of 14.7 and $8 \%$ for positioning accuracy are obtained for the north and east components, respectively, while a remarkable improvement of $37.3 \%$ is achieved for the vertical component. In terms of the BeiDou/GPS PPP solutions, the ECMWF-augmented PPP scenarios attain over 10\% improvements in 3D accuracy in all processing session lengths. These improvements totally come from the vertical component, whereas almost no enhancements are obtained in two horizontal components.
\end{abstract}

Keywords: Precise point positioning (PPP), BeiDou, Real-time, Tropospheric delay, European Centre of MediumRange Weather Forecasts (ECMWF), Convergence time, Positioning accuracy

\section{Introduction}

The precise point positioning technique, utilizing undifferenced carrier phase and code measurements and applying precise satellite orbit and clock corrections, is able to perform high-precision positioning with a single Global Navigation Satellite System (GNSS) receiver (Zumberge et al. 1997). In a standard PPP processing, the tropospheric delay parameters are usually estimated

\footnotetext{
*Correspondence: shjzhang@sgg.whu.edu.cn; rlliu@whu.edu.cn

${ }^{1}$ School of Geodesy and Geomatics, Wuhan University, Wuhan 430079,

China

${ }^{2}$ Water Resources and Hydropower Engineering, Wuhan University,

Wuhan 430072, China

Full list of author information is available at the end of the article
}

as unknowns with other parameters such as station coordinates, receiver clock offsets, and ambiguity parameters (Kouba and Héroux 2001). However, the parameters estimated in the PPP processing are highly correlated. In particular, according to previous studies, the convergence time for the PPP solution shows a high dependence on the estimation of the tropospheric delay parameters, which obstructs the fast convergence in PPP processing (Li et al. 2011). Hence, it is important to improve the tropospheric delay modeling and estimation to speed up the solution convergence for the PPP technique.

To solve this problem, Li et al. (2011) utilized the tropospheric delay information derived from a regional reference network to augment the real-time PPP (RT-PPP) 
instantaneous ambiguity resolution, which turned out to be effective in improving the convergence for the solution to an adequate accuracy level. Hadas et al. (2013) demonstrated the effectiveness of a regional tropospheric model for PPP in simulated RT-PPP as well. An enhancement of several centimeters in the vertical component and a few minutes in convergence time was found in their research. In addition, a regional tropospheric model retrieved from a continuously operating reference station (CORS) network was also introduced to augment the RTPPP solution by Shi et al. (2014). However, nearby reference stations are required for the regional tropospheric model augmentation method. Moreover, the approach is difficult to apply on a global scale.

Recently, numerical weather models (NWMs) have been developing rapidly. Initially, the tropospheric information derived from a GPS network was applied in the assimilation of NWM (Reigber et al. 2001). In return, accurate tropospheric information can be obtained from an NWM. Chen et al. (2011) evaluated tropospheric delays derived from the European Centre for MediumRange Weather Forecasts (ECMWF) and National Centers for Environmental Prediction (NCEP). Their results revealed the accuracy and feasibility of the tropospheric delays with ECMWF and NCEP data for navigation and positioning. Furthermore, Alves et al. (2016) used Saastamoinen model and numeric integration method to retrieve tropospheric delays from a regional NWM over South America and employed them in GPS PPP processing. Their experiment manifested an improvement of 7.2 in 3D RMS compared to the standard model. Lu et al. (2016) utilized the ECMWF data with a horizontal resolution of $1^{\circ} \times 1^{\circ}$ on 137 vertical model levels with the ray-tracing algorithm proposed by Zus et al. (2014) to retrieve the tropospheric delay parameters and apply them in the GNSS processing. The convergence time was shortened by $20.0,32.0$ and $25 \%$, and the positioning accuracy was improved by $2.5,12.1$ and $18.7 \%$ for the north, east and vertical components in their research.

The Chinese BeiDou Navigation Satellite System has been able to provide regional positioning, navigation and timing (PNT) since the end of 2012 and will provide full global navigation service by 2020 , according to the official announcement. The positioning accuracy of the BeiDou PPP is considered to be comparable to that of the GPS PPP, according to previous studies (Li et al. 2014, 2015). However, compared to the GPS PPP solution, a significantly longer convergence time is required for the BeiDou PPP solution due to insufficient satellite visibility and limited spatial geometry ( $\mathrm{Li}$ et al. 2015; Lu et al. 2016). To solve the problem, Lu et al. (2017) utilized the NCEP data on 26 isobaric levels with a horizontal resolution of $1^{\circ}$ and the ray-tracing algorithm developed by
Zus et al. (2014) to derive station-specific zenith delays, mapping function factors and other parameters. Their research showed a great improvement of 60 and 66.7\% in the convergence time for the east and vertical components, respectively, and an advancement of 40.1, 51.2 and $39.4 \%$ is obtained in positioning accuracy for the north, east and up components, respectively. However, the calculation procedures in their research, both the calculation of zenith delays by the ray-tracing algorithm and the estimation of mapping function factors, are quite complex and time-consuming.

In this study, we did not apply the ray-tracing algorithm and the NWM pressure level data with many isobaric levels introduced by $\mathrm{Lu}$ et al. (2016, 2017); instead, the Saastamoinen model is applied with the ECMWF surface level data, which only contains one level data related to the earth surface. The tropospheric delays derived from the ECMWF surface level data were then introduced into the PPP processing to augment the solutions. To verify the effectiveness of the introduced approach, both BeiDou-only and BeiDou/GPS standard PPP and the introduced ECMWF-augmented PPP scenarios over the same period and stations were processed. The augmentation performance is assessed in the case of convergence time and positioning accuracy.

In the following discussion, we first introduce the data used in our experiments in second section, including the ECMWF data and BeiDou observables. The experimental methodology is then described in third section. Fourth section presents the results and analysis and the summary and conclusion are given in final section.

\section{Data collection ECMWF data}

The ECMWF data utilized in this paper are derived from ERA-Interim dataset, which is the latest global atmospheric reanalysis produced by the ECMWF (Dee et al. 2011). The ERA-Interim provided gridded data, describing weather as well as ocean-wave and land-surface conditions. The gridded data is provided with a temporal resolution of $3 \mathrm{~h}$ for surface parameters and $6 \mathrm{~h}$ for upper-air parameters. For spatial resolution, the ERAInterim provides gridded data on surface level and up to 60 vertical pressure levels with a horizontal resolution from $0.125^{\circ}$ to $3^{\circ}$. Two types of data, in pressure level and surface level, are most commonly used to retrieve tropospheric delays and applied in GNSS positioning. The pressure level data usually contains many measurements corresponding to the vertical pressure on a grid for one meteorological parameter, in contrast, only one measurement is incorporated in the surface level data. Accordingly, the pressure level data is more extensive than the surface level data. Moreover, the derivation algorithm for 
tropospheric delays from the pressure level data is much more complicated and time-consuming than that from the surface level data (Zus et al. 2014). Consequently, two types of gridded data on surface level are utilized in this contribution. These data includes geopotential, pressure, 2-m dew point temperature and temperature with a horizontal resolution of $0.125^{\circ} \times 0.125^{\circ}$ and different temporal resolutions. The analysis data is provided with a 6-hourly resolution and the prediction data with a 3-hourly resolution. In particular, the prediction data is available before a measurement starts and able to be applied in a real-time positioning scenario. The dew point temperature is used to compute relative humidity requisite for troposphere delay calculation, because no available relative humidity is provided in surface level data.

\section{BeiDou data}

Thanks to the MGEX campaign initiated by IGS, a multiGNSS service of tracking, data collection and analysis is enabled by merging all available systems and signals, including GPS, GLONASS, BeiDou, Galileo, QZSS, and the space-based augmentation system (SBAS) (Montenbruck et al. 2017). As of March 2018, over 170 stations are capable of providing BeiDou observations in the MGEX network. 8 MGEX stations in Asia-Pacific area are selected in our experiment. They are capable of tracking more than 5 BeiDou satellites all day and providing BeiDou observables over the period from February 6 to March 8, 2016. The geographic distribution of the stations is shown in Fig. 1.

Orbit and clock products are available as part of the MGEX data analysis. Currently, three institutions provide orbit and clock products for the BeiDou constellation, including Center for Orbit Determination in Europe (CODE), GeoForschungsZentrum Potsdam (GFZ) and Wuhan University. In this contribution, we adopted 5 min MGEX ephemeris and 30 s clock products from GFZ (Deng et al. 2016; Uhlemann et al. 2015).

\section{PPP and processing strategy Standard PPP}

In a standard PPP, ionosphere-free combinations are commonly utilized as the processing observations. The antenna phase correction offset (PCO), phase wind-up, solid tide, earth rotation and relativistic effects are corrected by models (Kouba and Héroux 2001). The antenna phase correction variations (PCVs) are not corrected for BeiDou constellation owing to the absence of precise PCV correction model for BeiDou satellites. Ionospheric delays are eliminated by the ionosphere-free combinations. In the case of tropospheric delays, the zenith hydrostatic delays (ZHDs) can be computed precisely by a tropospheric model, while the zenith wet delays
(ZWDs) are difficult to be modeled and usually estimated as unknowns in the PPP processing. The observation equations can be expressed as follows:

$$
\begin{aligned}
& \Delta P_{\mathrm{C}}^{\mathrm{G}}=\rho_{0}+c\left(\delta t_{\mathrm{r}}^{\mathrm{G}}-\delta t^{\mathrm{s}, \mathrm{G}}\right)+m_{\mathrm{w}} \cdot \mathrm{ZWD}+\varepsilon_{P_{\mathrm{C}}} \\
& \Delta L_{\mathrm{C}}^{\mathrm{G}}=\rho_{0}+c\left(\delta t_{\mathrm{r}}^{\mathrm{G}}-\delta t^{\mathrm{s}, \mathrm{G}}\right)+m_{\mathrm{W}} \cdot \mathrm{ZWD}+B_{\mathrm{C}}+\varepsilon_{L_{\mathrm{C}}}
\end{aligned}
$$

where the superscript $G$ is the satellite system (GPS/BeiDou); $\Delta P_{C}, \Delta L_{C}$ are the pseudo-range and phase observations of ionosphere-free combinations that remove the slant hydrostatic delays, respectively; $\rho_{0}$ is the geometric range from receiver to satellite; $\delta t_{\mathrm{r}}, \delta t^{\mathrm{s}}$ denote the receiver clock bias and satellite clock bias, respectively; $c$ is the light speed in vacuum; $m_{\mathrm{w}}$ is the wet mapping function and ZWD is the zenith wet delay; $B_{C}$ is the ambiguity for ionosphere-free observations; $\varepsilon_{P_{\mathrm{C}}}, \varepsilon_{L_{\mathrm{C}}}$ are pseudo-range and phase measurement noises, respectively. In a combined processing of BeiDou/GPS observations, the intersystem biases (ISBs) have to be estimated (Lu et al. 2016).

Accordingly, the estimated parameters for this PPP processing can be expressed as follows:

$$
X_{e}=\left[\Delta X, \delta t_{r}, Z W D,(I S B), B_{C}\right]^{T}
$$

where $\Delta \boldsymbol{X}$ represents the vector of receiver coordinate residuals with respect to the priori position which is adopted in linearization; $\boldsymbol{Z W D}$ is the zenith wet delay; $I S B$ denotes the inter-system bias, $\delta \boldsymbol{t}_{\boldsymbol{r}}$ represents the receiver clock error and $\boldsymbol{B}_{C}$ stands for the vector of ambiguities.

Specific processing strategy for a standard PPP will be described in "PPP processing strategy" section.

\section{ECMWF-augmented PPP}

The zenith wet delays derived from ECMWF surface level data, were applied as a priori values to the PPP processing and a corresponding constraint was added for the estimation of ZWD residuals. We term this PPP method as "ECMWF-augmented PPP". In the following sections, the derivation approach for ZWDs from ECMWF meteorological parameters on surface level as well as the way they are applied in an ECMWF-augmented PPP processing is described.

\section{Tropospheric wet delay derived from ECMWF}

The ERA-Interim data are gridded and provided with a 3-hourly or 6-hourly resolution. Therefore, an interpolation on time for these meteorological parameters is necessary to match the PPP sampling rate. Then, a computation for the zenith delays above the grids is required. 


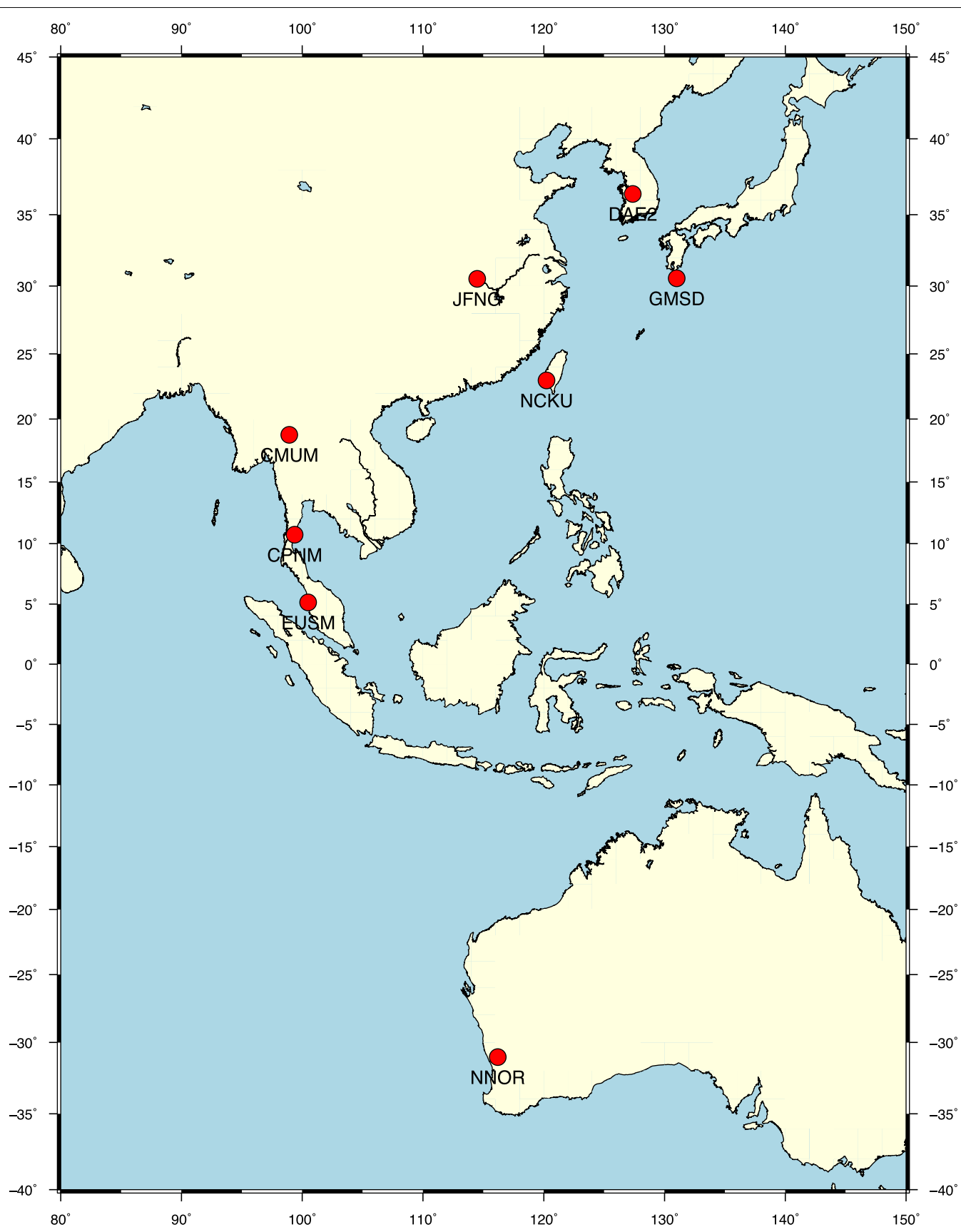

Fig. 1 The geographic distribution of stations from the MGEX network

Moreover, a spatial interpolation or fitting should be implemented to acquire the station-specific zenith delays from the grids.

Firstly, a linear interpolation is implemented on the meteorological parameters above the grids from the ECMWF data available moments to the observable sampling epoches. Secondly, the zenith wet delays above the grids are computed from these meteorological parameters using Saastamoinen model (Saastamoinen 1972; Davis et al. 1985):

$$
\begin{aligned}
& \mathrm{ZWD}=\frac{0.0022768 e\left(\frac{1255.0}{T}+0.05\right)}{f(\phi, h)} \\
& e=r h \cdot 6.112 \cdot \exp \left(\frac{17.62 t}{243.12+t}\right)
\end{aligned}
$$




$$
f(\phi, h)=1-0.00266 \cos 2 \phi-0.00028 h
$$

where $e$ is the vapor pressure in millibar; $T$ and $t$ are the surface temperature in $\mathrm{K}$ and ${ }^{\circ} \mathrm{C}$, respectively (Jarraud 2008); $\phi$ denotes the latitude in ${ }^{\circ}$; and $h$ is the altitude above the ellipsoid surface in $\mathrm{km}$; $r h$ is the relative humidity in percent and can be computed by the dew point temperature and the temperature (Alduchov and Eskridge 1996):

$$
r h=100 \cdot \frac{\exp \left(\frac{17.625 \mathrm{TD}}{243.04+\mathrm{TD}}\right)}{\exp \left(\frac{17.625 T}{243.04+T}\right)}
$$

where TD represents the dew point temperature; $T$ is the temperature in ${ }^{\circ} \mathrm{C}$. It is noteworthy that these meteorological parameters provided in geopotential must be converted to those in altitude before they are applied into the Saastamoinen model.

Thirdly, a spatial interpolation was implemented to obtain the zenith delays above the stations from the grids. These grids can be regarded as virtual reference stations which only provide zenith tropospheric information. A spatial interpolation model called H1QX1 was adopted. The H1QX1 model is considered to be more convenient for computation and its precision is equivalent when compared to another spatial interpolation model named MLCM or H1QM3, according to previous studies (Zhang et al. 2013; Li et al. 2011). Two regression parameters and height differences from stations to grids are adequate for the H1QX1 model. This model can be expressed as follows:

$$
\left[\begin{array}{c}
\mathrm{ZWD}_{1} \\
\mathrm{ZWD}_{2} \\
\vdots \\
\mathrm{ZWD}_{n}
\end{array}\right]=\left[\begin{array}{cc}
1 & H_{1} \\
1 & H_{2} \\
\vdots & \vdots \\
1 & H_{n}
\end{array}\right]\left[\begin{array}{l}
a_{0} \\
a_{1}
\end{array}\right]
$$

where $H_{\mathrm{n}}$ denotes the altitude differences from the reference grids to the station; $a_{\mathrm{n}}$ is the regression parameters. The equation can be expressed in vector form as follows:

$$
Z W D=H \cdot a
$$

The regression parameter vector $\boldsymbol{a}$ is determined by a least-squares estimation, which can be expressed by Eq. (10):

$$
\boldsymbol{a}=\left(\boldsymbol{H}^{T} \cdot \boldsymbol{P} \cdot \boldsymbol{H}\right)^{-1} \cdot \boldsymbol{H}^{T} \cdot \boldsymbol{P} \cdot Z W D
$$

where $\boldsymbol{P}$ is the weight matrix, and the weights are determined by the anti-distance method according to the distances between neighbor grids to the station.
The station-specific ZWD can be computed by the following equation:

$$
\mathrm{ZWD}_{\mathrm{s}}=e^{T} \cdot a
$$

where $\boldsymbol{e}$ is a unit vector and can be written as $\left[\begin{array}{ll}1 & 0\end{array}\right]^{T}$.

The H1QX1 model performs best in terms of interpolation precision when the number of reference stations is 9 or 10 (Zhang et al. 2013). Therefore, the ZWDs on 9 neighbor grids were applied in the interpolation procedure. Theoretically, the distances between these neighbor grids to the station vary from 0.0 for the nearest to $29.4 \mathrm{~km}$ for the furthest grid.

\section{ECMWF-augmented PPP method}

The ECMWF-augmented PPP method is based on the traditional PPP method. Different from the traditional PPP, the ECMWF-augmented method introduces ZWDs from ECMWF parameters on surface level as virtual observations. The observation equations then can be expressed as follows:

$$
\begin{aligned}
& \Delta P_{\mathrm{C}}=\rho_{0}+c\left(\delta t_{\mathrm{r}}-\delta t^{\mathrm{s}}\right)+m_{\mathrm{w}} \cdot \Delta \mathrm{ZWD}+\varepsilon_{P_{\mathrm{C}}} \\
& \Delta L_{\mathrm{C}}=\rho_{0}+c\left(\delta t_{\mathrm{r}}-\delta t^{\mathrm{s}}\right)+m_{\mathrm{w}} \cdot \Delta \mathrm{ZWD}+B_{\mathrm{C}}+\varepsilon_{L_{\mathrm{C}}}
\end{aligned}
$$

$$
\Delta \mathrm{ZWD}=0.0, \mathrm{QZWD}_{\mathrm{E}}
$$

where $\triangle Z W D$ is the residual of ZWD to estimate. Strictly, $\triangle Z W D$ in formula (14) should be set to the mean bias of the derived ZWDs compared to real values. The mean bias is about $0.1 \mathrm{~mm}$ for ECMWF-derived ZTDs according to previous studies and can be ignored (Chen et al. 2011). QZWDE is the stochastic model of ECMWFderived ZWDs and was given by Chen as well (Chen et al. 2011). $\Delta P_{C}, \Delta L_{C}$ are the ionosphere-free pseudo-range and phase observations that remove the slant hydrostatic delays and ECMWF-derived wet delays, respectively.

The ECMWF-derived ZWDs are introduced as a priori corrections on an epoch-wise basis; hence, the estimated parameters in ECMWF-augmented PPP can be expressed:

$$
X_{e}=\left[\Delta X, \delta t_{r}, \Delta Z W D,(I S B), B_{C}\right]^{T}
$$

Analysis and prediction data described in "ECMWF data" section, are introduced into the ECMWF-augmented PPP method. The prediction data was used to imitate the real-time PPP processing as the software for computing real-time BeiDou satellite orbits and clocks is still under development at this point. The analysis data are utilized to verify the PPP processing results. 


\section{PPP processing strategy}

PPPBox, a GNSS processing software based on GPS Toolkit, was modified and developed by the authors to process the multi-GNSS data in these aforementioned PPP approaches. For brevity, the ECMWF-augmented PPP using analysis data is simplified into "ECMWF-A PPP", accordingly, the augmented PPP with prediction data is reduced to "ECMWF-P PPP". The ZHDs for three PPP methods are given by the same exponential function from Niell troposphere model (Niell 1996). The ZWD is estimated as an unknown parameter in a standard PPP, while in the proposed augmented PPP approach, residual ZWD is under estimation in regard to a priori value derived from ECMWF. Then, an extra equation, working as a virtual observation with respect to the variance of derived ZWDs, is added to the observation equations. Niell Mapping Function (NMF) is adopted for the tropospheric mapping function (Niell 1996). For the sake of brevity, other identical items are shown in Table 1.

\section{Results and analysis}

In this section, we compare the performance of the standard PPP method and ECMWF-augmented PPP methods using analysis and prediction data in the case of the convergence time and positioning accuracy. The convergence time is defined as the $3 \mathrm{D}$ positioning accuracy achieves $10 \mathrm{~cm}$.

The positioning error series of BeiDou-only PPP methods on station NNOR at February 15, 2016 (DOY 44 of 2016) is taken as an example and displayed in Fig. 2. Blue represents the standard PPP solution, green and red stand for the ECMWF-augmented PPP solutions with analysis and prediction data, respectively.

The positioning error series of BeiDou-only PPP methods on station NNOR at February 15, 2016 (DOY 44) is taken as an example and displayed in Fig. 2. Blue represents the standard PPP solution, green and red stand for the ECMWF-augmented PPP solutions with analysis and prediction data, respectively.

In the north component, the positioning error curves for three PPP solutions coincide well with each other. Similar situation emerges in the east component, indicating that the convergence time and positioning accuracy are comparable for three PPP solutions in the horizontal components. Specifically, it takes approximately $0.5 \mathrm{~h}$ for the convergence of three PPP solutions in the horizontal component. For the vertical component, the fluctuation in the standard PPP positioning error curve is significantly larger than the two ECMWF-augmented PPP solutions at the beginning of processing. It takes approximately $1.5 \mathrm{~h}$ for the standard PPP solution to get converged, in comparison with $0.5 \mathrm{~h}$ required for the two ECMWF-augmented PPP solutions. The ECMWFA PPP solution achieves the highest positioning accuracy when all solutions get converged, then follows the ECMWF-P PPP solution, the standard PPP solution performs the worst. In general, the augmented PPP solution using analysis data performs slightly better than the one using prediction data. In summary, remarkable improvements can be noted in the two ECMWF-augmented PPP solutions compared to the standard PPP solution for the convergence time and positioning accuracy in vertical component, while small improvements are observed in two horizontal components. This is attributed to the large correlation between ZTD and vertical coordinate. The accuracy of vertical coordinate will definitely get improved because the wet delays are properly constrained in vertical, whereas for the two horizontal coordinates, only small improvements can be attained.

Figures 3 and 4 illustrate the 3D RMS values of positioning results for BeiDou-only and BeiDou/GPS PPP

Table 1 PPP processing strategies

\begin{tabular}{|c|c|c|c|}
\hline & Standard PPP & ECMWF-A PPP & ECMWF-P PPP \\
\hline Sampling rate(s) & 30 & & \\
\hline Cutting-off angles & $10^{\circ}$ & & \\
\hline Observations & Ionosphere-free combinations & & \\
\hline Antenna & igs08.atx & & \\
\hline Estimator & Forward-only Kalman filter & & \\
\hline Ambiguity & Float & & \\
\hline Troposphere ZHD & Niell model & & \\
\hline Troposphere ZWD & $\begin{array}{l}\text { Estimation of ZWD [random } \\
\text { walk process } 10 \mathrm{~mm} / \mathrm{sqrt}(\mathrm{h})]\end{array}$ & $\begin{array}{l}\text { ECMWF analysis data and estimation of } \\
\text { residual ZWD [random walk process } \\
10 \mathrm{~mm} / \mathrm{sqrt}(\mathrm{h})]\end{array}$ & $\begin{array}{l}\text { ECMWF prediction data and estima- } \\
\text { tion of residual ZWD [random walk } \\
\text { process } 10 \mathrm{~mm} / \mathrm{sqrt}(\mathrm{h})]\end{array}$ \\
\hline Troposphere mapping function & \multicolumn{3}{|l|}{ Niell mapping function } \\
\hline Multi-GNSS satellite clock and orbit & \multicolumn{3}{|c|}{ MGEX clock and orbit from GFZ (Deng et al. 2016) } \\
\hline
\end{tabular}



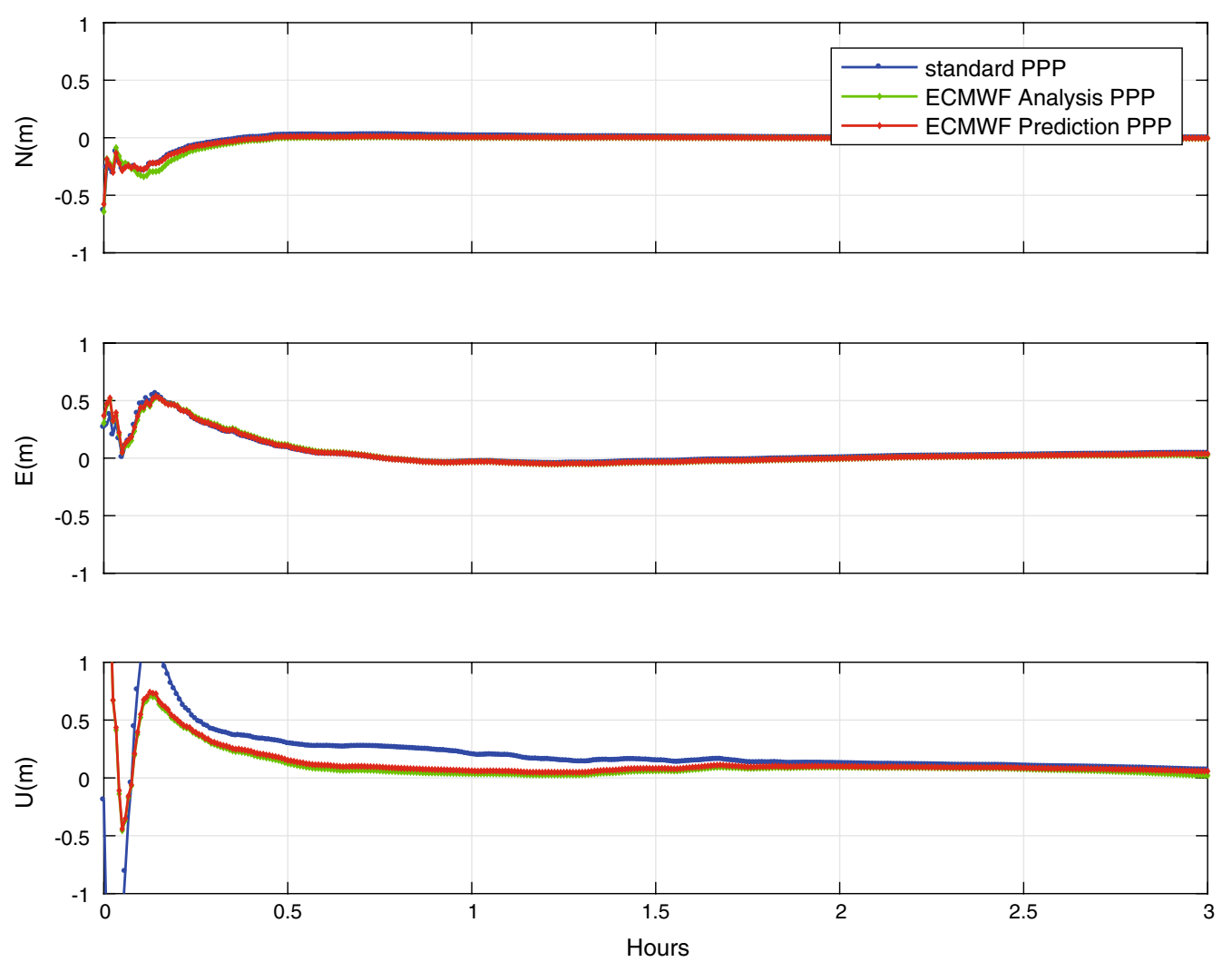

Fig. 2 BeiDou-only PPP solutions at station NNOR on February 15, 2016 [day of year (DOY) 44 of 2016]. Blue denotes the standard PPP solution, green represents the ECMWF-augmented PPP solution with analysis data, and red stands for the ECMWF-augmented PPP solution with prediction data

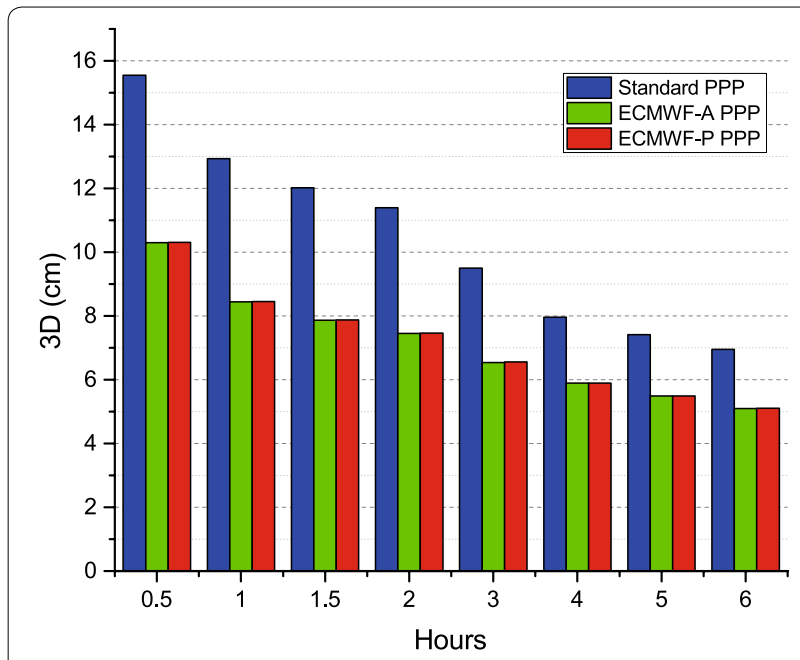

Fig. 3 The 3D RMS values of BeiDou-only PPP results after the processing of different session length $(0.5,1,1.5,2,3,4,5,6 \mathrm{~h})$ from the MGEX stations exhibited in Fig. 1 over a sample period from February 6 to March 8, 2016. Blue denotes the standard PPP solution, green and red represent the ECMWF-augmented PPP solution with analysis and prediction data, respectively solutions after the processing of different session length. The case is the same as it was mentioned earlier.

As can be noticed in two figures, the positioning accuracy for three PPP solutions is highly correlated to the session length. The positioning accuracy improves as the session length gets longer. In the BeiDou-only PPP solutions, it takes generally $2.7 \mathrm{~h}$ for the standard PPP solution to get converged to a 3D accuracy of $10 \mathrm{~cm}$, in contrast, only $32 \mathrm{~min}$ is required for the two ECMWF-augmented PPP solutions, representing a remarkable advancement of $80.6 \%$. After a 2.7 -h session length required for the standard PPP solution to get converged, the positioning accuracy for the two ECMWFaugmented PPP results are $6.6 \mathrm{~cm}$, corresponding to an improvement of $34 \%$. In addition, the $3 \mathrm{D}$ positioning accuracy for the two ECMWF-augmented PPP solutions are comparable in different session length. Regarding the BeiDou/GPS PPP results displayed in Fig. 4, higher positioning accuracy and shorter convergence time are attained in the BeiDou/GPS PPP solutions in contrast to the BeiDou-only PPP solutions, due to the increased satellites from an additional constellation. Nonetheless, 


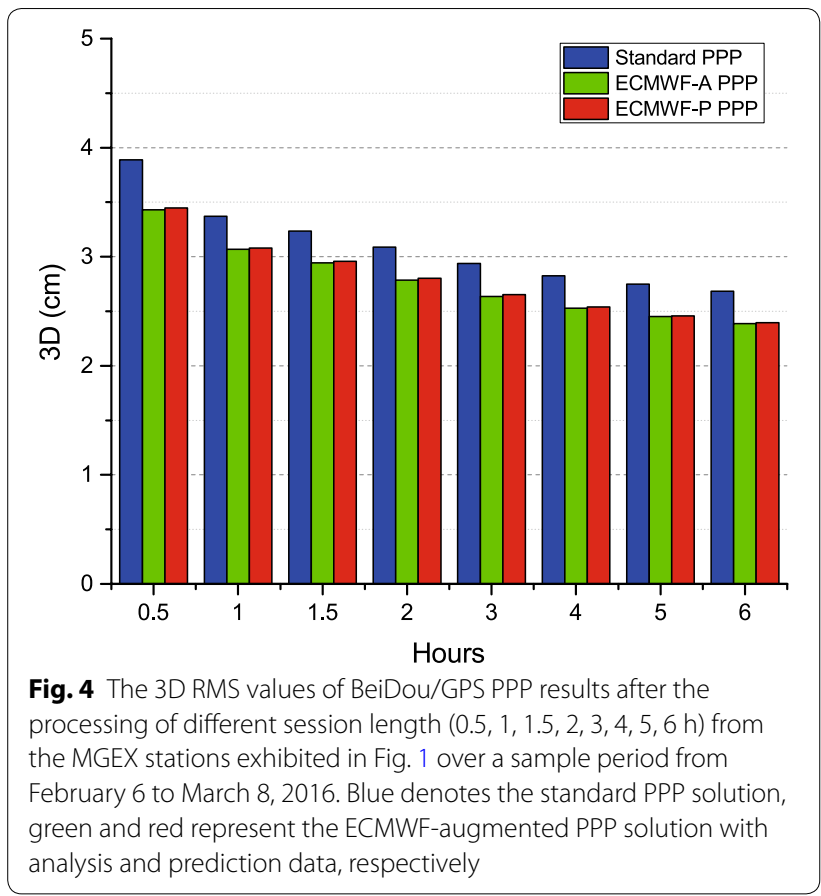

the improvements in positioning accuracy brought by the introduced augmented method in the BeiDou/GPS PPP results do not reveal as dramatic as that in the BeiDouonly PPP solutions. Enhancements of over 10\% on 3D positioning accuracy are obtained by the introduced augmented approaches in the BeiDou/GPS PPP solutions, compared to over $30 \%$ in the BeiDou-only PPP case. The increased number of visible GNSS satellites and stronger satellite geometry strength are mainly responsible for this. Furthermore, the positioning accuracy is nearly similar in two augmented BeiDou/GPS PPP solutions. The same case appears in the BeiDou-only PPP solutions as well.

To investigate the separate benefits on the improvements of positioning accuracy for each component, the RMS values of the BeiDou-only and BeiDou/GPS PPP results for the north, east and up component after the processing of different session length. Same case has been mentioned earlier (Figs. 5, 6).

In the BeiDou-only PPP scenarios, one can notice that limited improvements are obtained by the two ECMWFaugmented PPP solutions. Specifically, after a session length of $2.7 \mathrm{~h}$, the positioning accuracy is $1.4 \mathrm{~cm}$ for the two ECMWF-augmented PPP solutions in the north component, in comparison with $1.7 \mathrm{~cm}$ for that of the standard PPP solution, revealing an improvement of $14.7 \%$. Meanwhile, a positioning accuracy of $2.3 \mathrm{~cm}$ can be achieved for the two augmented PPP solutions in the east component, in contrast to $2.5 \mathrm{~cm}$ for that of the
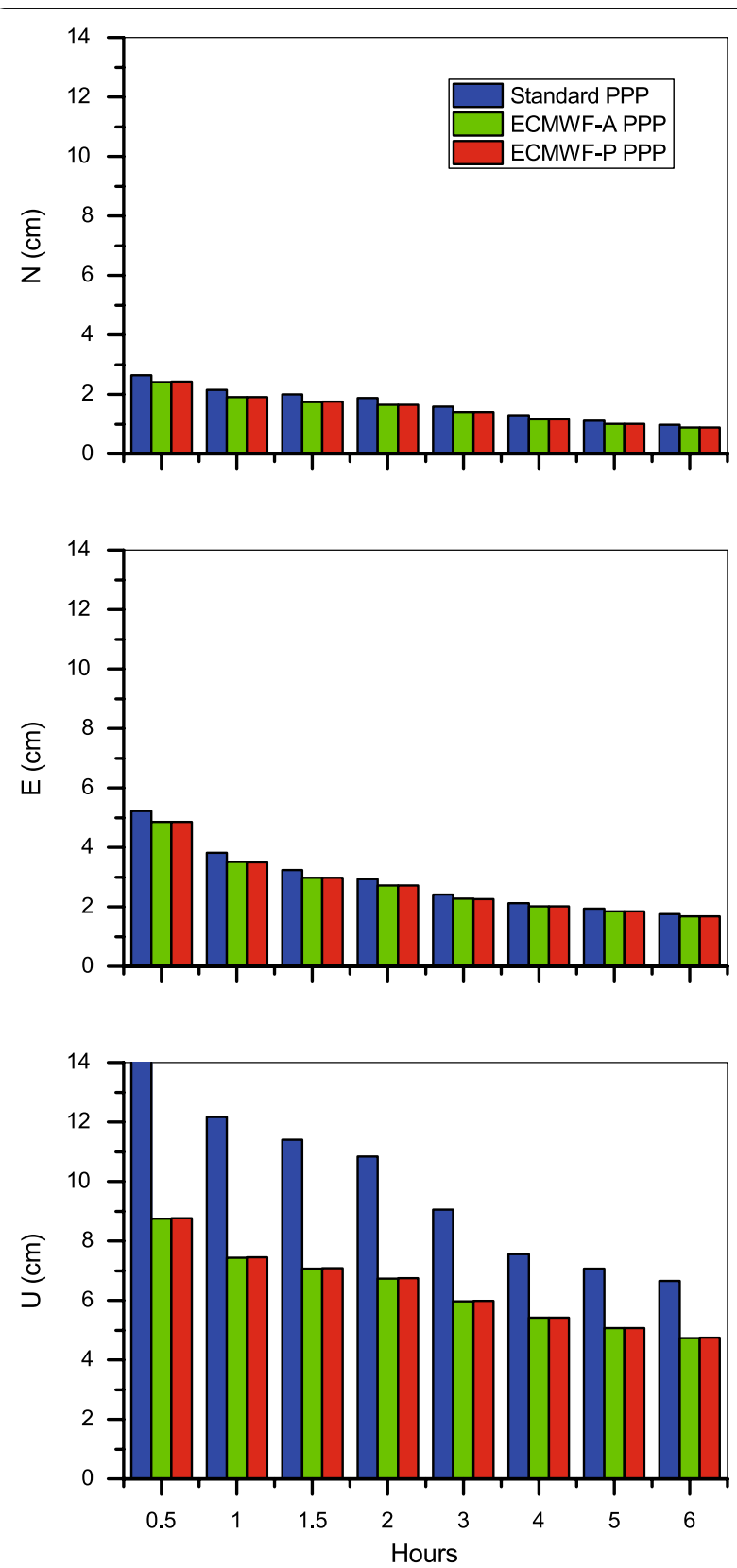

Fig. 5 RMS values of BeiDou-only results for the north/east/up components after the processing of different session length $(0.5,1$, $1.5,2,3,4,5,6 \mathrm{~h}$ ) from the BeiDou stations in Fig. 1 over a sample period from February 6 to March 8, 2016. Blue denotes the standard PPP solution, green and red are the ECMWF-augmented PPP solution with analysis and prediction data, respectively

standard PPP solution, an improvement of $8.0 \%$. With respect to the vertical component, significant improvements are obtained by the two augmented PPP solutions compared to the standard PPP solution at all session lengths. Moreover, after a 2.7-h session length, the positioning accuracy achieves $6.1 \mathrm{~cm}$ for the two augmented 

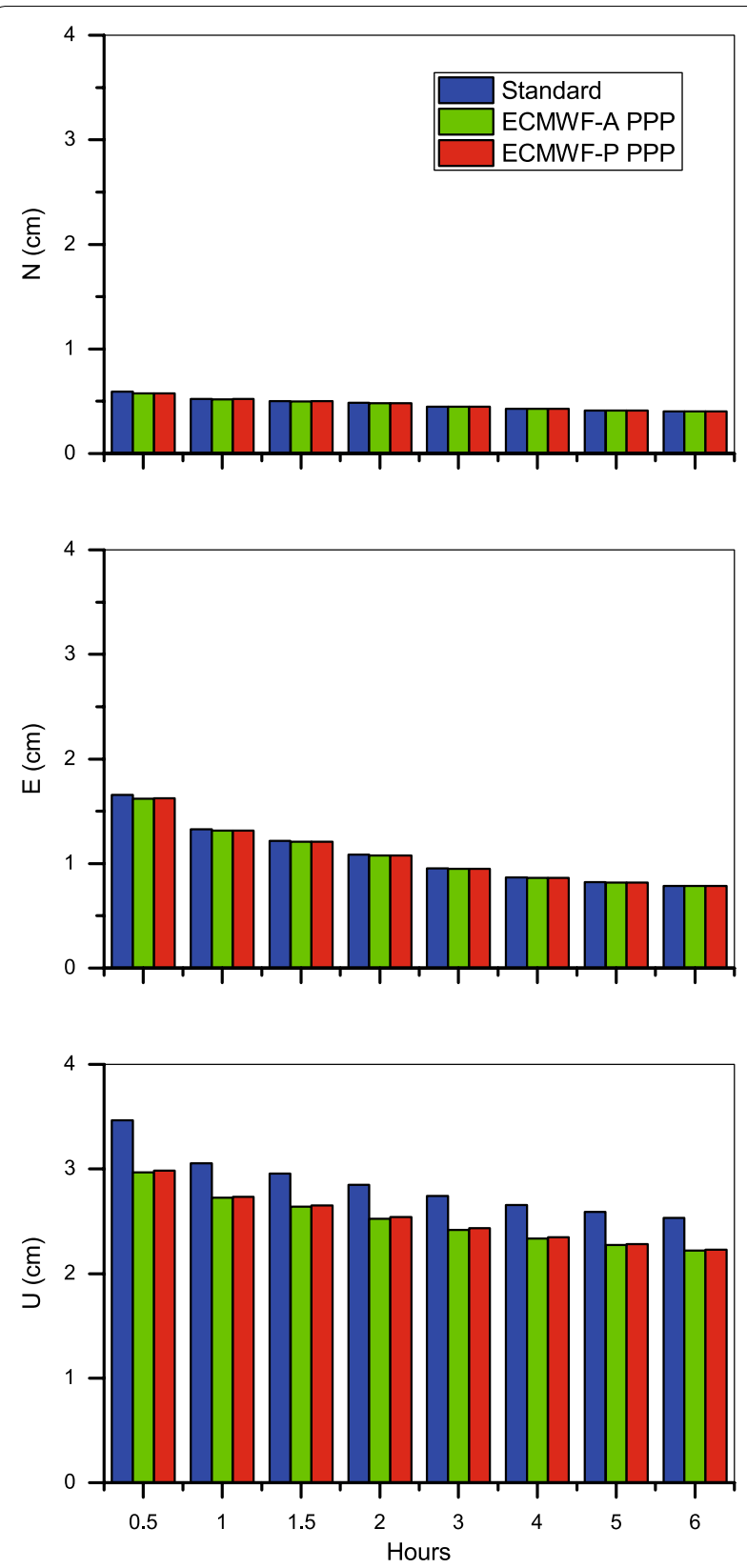

Fig. 6 RMS values of BeiDou/GPS PPP results for the north/east/ up components after the processing of different session length (0.5, $1,1.5,2,3,4,5,6$ h) from the BeiDou stations in Fig. 1 over a sample period from February 6 to March 8, 2016. Blue stands for the standard PPP solution, green and red denote the ECMWF-augmented PPP solution with analysis and prediction data, respectively
PPP solutions in the vertical component, in comparison with $9.5 \mathrm{~cm}$ for that of the standard PPP solution, representing an improvement of $37.3 \%$. When comparing the two augmented PPP solutions with each other, it demonstrates that similar enhancements are realized with respect to the standard PPP solution in all components.
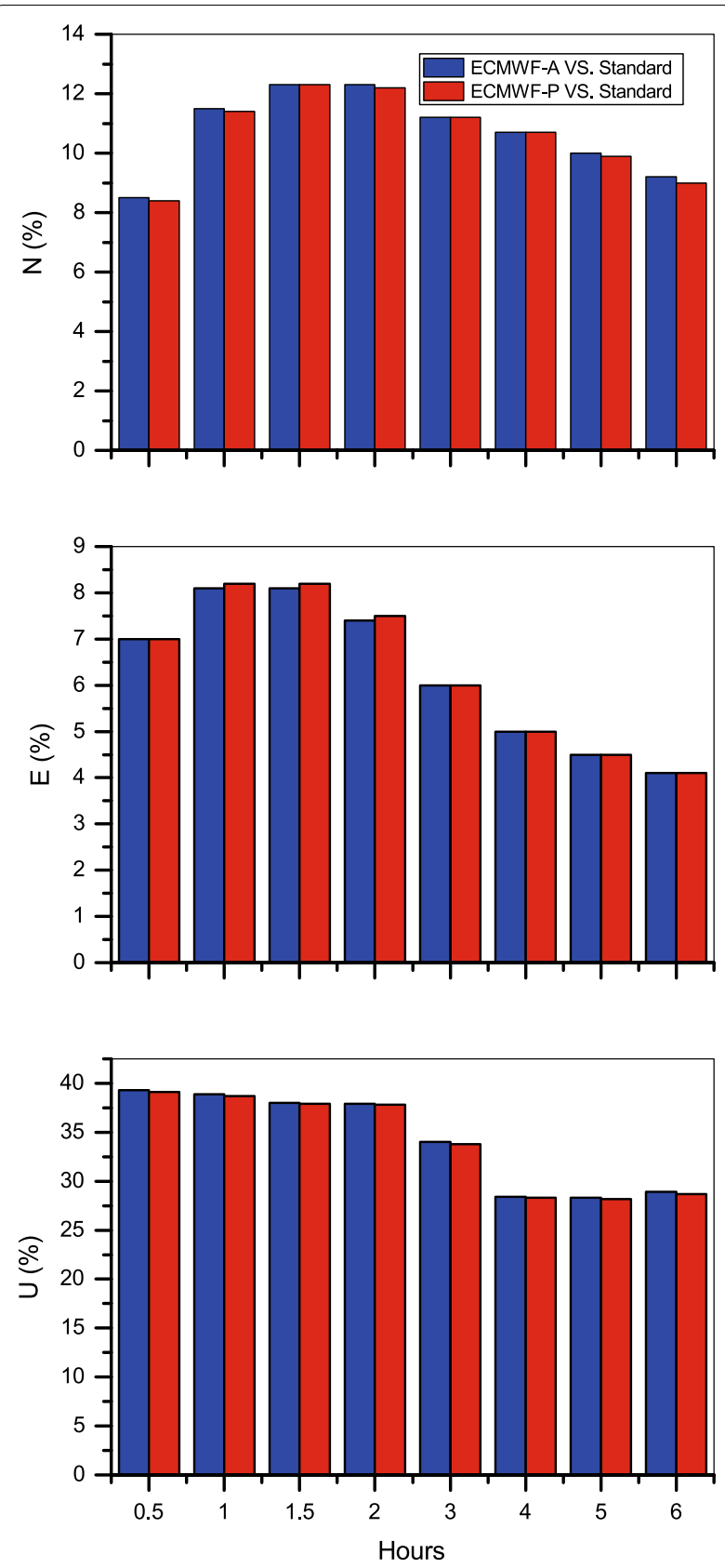

Fig. 7 Specific increased ratios of the two ECMWF-augmented PPP results compared to standard PPP results in BeiDou-only at different session length. The improvements in percentage for ECMWF-augmented PPP results using analysis and prediction data are displayed in blue and red, respectively

Specific increased ratios of the two ECMWF-augmented PPP results in comparison with the standard PPP result at different session length are shown in Fig. 7. The improvements in percentage for ECMWF-augmented PPP results using analysis and prediction data versus the standard PPP results are displayed in blue and red, respectively. 

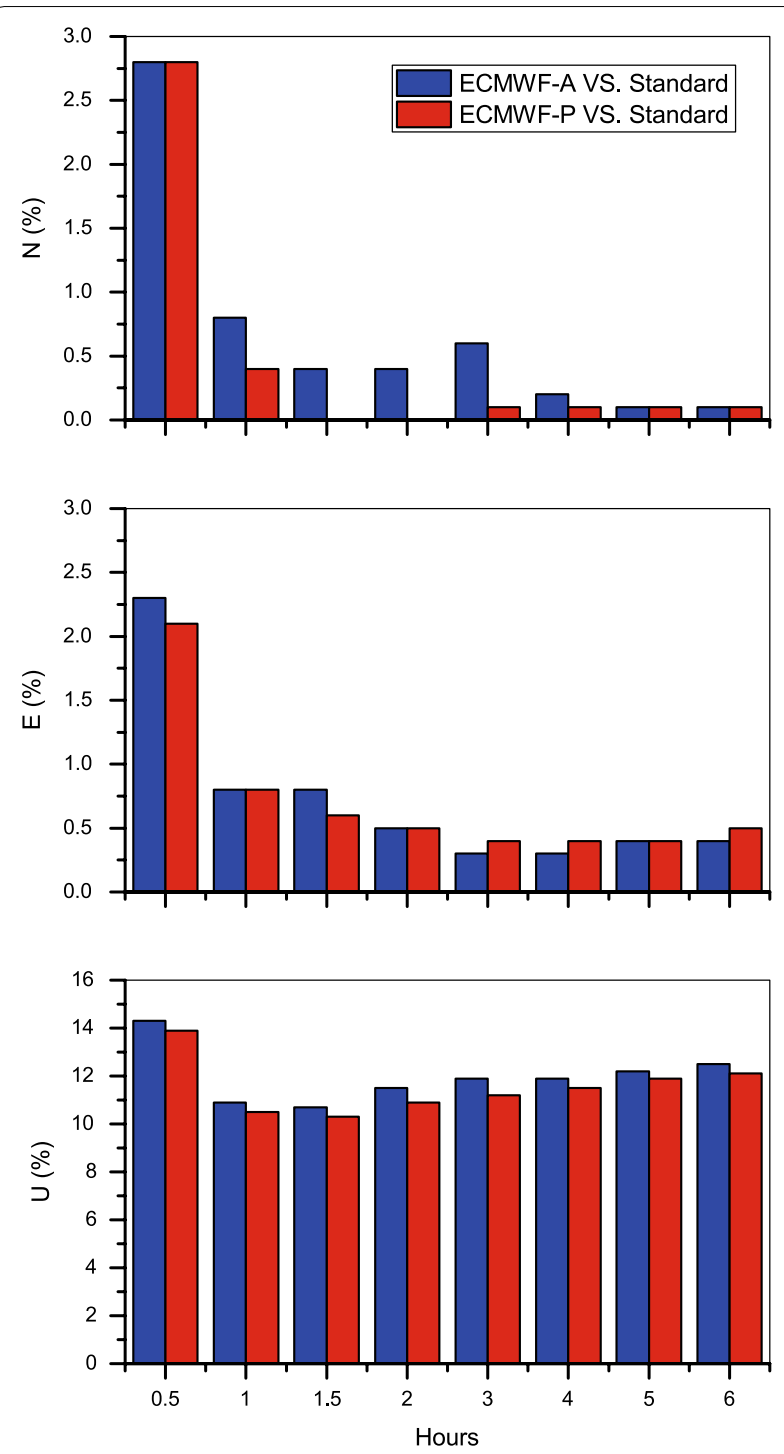

Fig. 8 Specific increased ratios of the two ECMWF-augmented PPP results compared to standard PPP results in BeiDou/GPS PPP at different session length. Blue and red stand for the improvements in percentage for ECMWF-augmented PPP results using analysis and prediction data, respectively

In the BeiDou/GPS PPP solutions, few contributions are discovered in north and east components and almost all contributions to 3D positioning accuracy come from the up component. Explicit ratios are displayed in Fig. 8, as can be noted, less than $3 \%$ improvements in two augmented BeiDou/GPS PPP methods in different session length are realized in two horizontal components in contrast to the standard BeiDou/GPS PPP scenario. In vertical component, over $10 \%$ enhancement is obtained in different session length. It is noteworthy that the horizontal positioning accuracy is improved by the augmented approach in the BeiDou-only PPP scenarios, but the improvements are not distinct in the BeiDou/ GPS PPP cases. Similar case can be observed in the vertical component: the increased ratios brought by the augmented method in the BeiDou-only PPP cases are larger than that in the BeiDou/GPS PPP scenarios. As we all know, there is a large correlation between tropospheric delay, vertical coordinate and receiver clock. The correlation is resolved much faster in a standard BeiDou/GPS PPP case than in a standard BeiDou-only scenario due to better geometry and additional satellites. The ZWD constrains actually accelerate the correlation resolving process in the augmentation method. This explains why the augmentation method performs better in the BeiDouonly PPP cases than in the BeiDou/GPS PPP scenarios. The positioning accuracy of two augmented BeiDou/GPS PPP methods are comparable in all directions, the same as can be observed in the BeiDou-only PPP approaches.

\section{Summary and conclusion}

In this study, the tropospheric information derived from the ECMWF analysis and prediction data were applied to the simulated real-time BeiDou-only and BeiDou/GPS PPP to augment the solutions. Two ECMWF-augmented PPP solutions are carried out in comparison with the standard PPP solution to evaluate the performance of the introduced ECMWF-augmented approach in terms of convergence time and positioning accuracy.

It takes approximately $2.7 \mathrm{~h}$ for the BeiDou-only standard PPP solution to get converged to a $3 \mathrm{D}$ accuracy of $10 \mathrm{~cm}$, in comparison with only $32 \mathrm{~min}$ cost for the two ECMWF-augmented BeiDou PPP solutions, representing a remarkable advancement of $80.6 \%$. When the standard PPP solution gets converged, the two ECMWF-augmented PPP methods obtain a 3D accuracy of about $6.6 \mathrm{~cm}$, corresponding to an enhancement of $34 \%$. As for the three separated components, only slight improvements for north and east components can be realized. Specifically, after a 2.7-h session length, the positioning accuracy for the two ECMWFaugmented BeiDou-only PPP solutions is 1.4 and $2.3 \mathrm{~cm}$ in the north and east component, respectively, with respect to $1.7 \mathrm{~cm}$ and 2.5 for the standard BeiDou PPP solution, corresponding to improvements of 14 and $8 \%$, respectively. With respect to the vertical component, the positioning accuracy improves from 9.5 to $6.1 \mathrm{~cm}$ when comparing the standard PPP solution with the two ECMWF-augmented PPP solutions in BeiDouonly scenario, exhibiting a significant improvement of $37.3 \%$. In addition to the BeiDou/GPS PPP solutions, all three PPP solutions get converged in a 30 -min session length. The two augmented PPP solutions attain about $10 \%$ higher $3 \mathrm{D}$ accuracy in the same session length. The 
improvements totally come from the vertical component, and no improvements are observed in the north and east component. The augmentation works more effectively in a BeiDou-only PPP scenario than in a BeiDou/GPS PPP case because the correlation between tropospheric delay and vertical coordinate is resolved much faster in the BeiDou/GPS PPP case due to better geometry and additional satellites. Meanwhile, the comparisons of two ECMWF-augmented methods in BeiDou-only and BeiDou/GPS PPP scenarios reveal that the one using prediction data performs as well as the one using analysis data. This indicates that the ECMWF prediction data is effective to be introduced in the real-time BeiDou-only and BeiDou/GPS PPP scenarios. In summary, the proposed ECMWF-augmented PPP approach is effective to improve the positioning accuracy and shorten the convergence time for the realtime PPP application.

\begin{abstract}
Abbreviations
GPS: Global Positioning System; PPP: precise point positioning; ECWMF: European Centre of Medium-Range Weather Forecasts; IGS: International GNSS Service; MGEX: Multi-GNSS Experiments; 3D: three dimensional; GNSS: Global Navigation Satellite System; RT-PPP: real-time precise point positioning; CORS: continuous operating reference station; NWM: numerical weather model; NCEP: National Centers for Environmental Prediction; PNT: positioning, navigation and timing; QZSS: Quasi-Zenith Satellite System; SBAS: space-based augmentation system; CODE: Center for Orbit Determination in Europe; GFZ: GeoForschungsZentrum Potsdam; PCO: phase correction offset; PCV: phase correction variation; ZWD: zenith wet delay; ZHD: zenith hydrostatic delay; ZTD: zenith total delay; STD: slant total delay.
\end{abstract}

\section{Authors' contributions}

All the authors contributed to the design of the proposed. SZ came up with the idea of the constrain method. LZ, WW and KZ achieved the proposed method, KZ carried out the experiments and drafted the manuscript. RL and JW participated in the experimental analysis. All authors read and approved the final manuscript.

\section{Author details}

${ }^{1}$ School of Geodesy and Geomatics, Wuhan University, Wuhan 430079 China. ${ }^{2}$ Water Resources and Hydropower Engineering, Wuhan University, Wuhan 430072, China. ${ }^{3}$ School of Civil and Environmental Engineering, The University of New South Wales, Sydney, NSW 2052, Australia.

\section{Acknowledgements}

We greatly appreciate the ECMWF for the online provision of the high spatial and time resolution meteorological data. We also thank the IGS for providing the BeiDou observations and the GFZ for the multi-constellation orbit and clock products.

\section{Competing interests}

The authors declare that they have no competing interests.

\section{Availability of data and materials}

The BeiDou observations, MGEX ephemeris and clock products from IGS are available are available at CDDIS FTP site: ftp://cddis.eosdis.nasa.gov.

The ECMWF data can be downloaded in ERA-Interim dataset: http://apps. ecmwf.int/archive-catalogue/?class=ei.

Ethics approval and consent to participate Not applicable.
Funding

None.

\section{Publisher's Note}

Springer Nature remains neutral with regard to jurisdictional claims in published maps and institutional affiliations.

Received: 13 January 2018 Accepted: 8 June 2018

Published online: 03 July 2018

\section{References}

Alduchov OA, Eskridge RE (1996) Improved Magnus form approximation of saturation vapor pressure. J Appl Meteorol 35(4):601-609

Alves DBM, Sapucci LF, Marques HA, de Souza EM, Gouveia TAF, Magário JA (2016) Using a regional numerical weather prediction model for GNSS positioning over Brazil. GPS Solut 20(4):677-685

Chen Q, Song S, Heise S, Liou YA, Zhu W, Zhao J (2011) Assessment of ZTD derived from ECMWF/NCEP data with GPS ZTD over China. GPS Solut 15(4):415-425

Davis JL, Herring TA, Shapiro I, Rogers AE, Elgened G (1985) Geodesy by interferometry: effects of atmospheric modeling errors on estimates of base line length. Radio Sci 20:1593-1607

Dee DP, Uppala SM, Simmons AJ, Berrisford P, Poli P, Kobayashi S et al (2011) The ERA-Interim reanalysis: configuration and performance of the data assimilation system. Q J R Meteorol Soc 137:553-597. https://doi. org/10.1002/qj.828

Deng Z, Fritsche M, Nischan T, Bradke M (2016) Multi-GNSS ultra rapid orbit-, clock- \& EOP-product series. http://doi.org/10.5880/GFZ.1.1.2016.003

Hadas T, Kaplon J, Bosy J, Sierny J, Wilgan K (2013) Near-real-time regional troposphere models for the GNSS precise point positioning technique. Meas Sci Technol 24:055003

Jarraud M (2008) Guide to meteorological instruments and methods of observation (WMO-No. 8). World Meteorological Organisation, Geneva

Kouba J, Héroux P (2001) Precise point positioning using IGS orbit and clock products. GPS Solut 5(2):12-28. https://doi.org/10.1007/PL00012883

Li X, Zhang X, Ge M (2011) Regional reference network augmented precise point positioning for instantaneous ambiguity resolution. J Geod 85(3):151-158

Li M, Qu L, Zhao Q, Guo J, Su X, Li X (2014) Precise point positioning with the BeiDou navigation satellite system. Sensors 14:927-943

Li X, Ge M, Dai X, Ren X, Fritsche M, Wickert J, Schuh H (2015) Accuracy and reliability of multi-GNSS real-time precise positioning: GPS, GLONASS, BeiDou, and Galileo. J Geod 89(6):607-635. https://doi.org/10.1007/s0019 0-015-0802-8

Lu C, Zus F, Heinkelmann R, Dick G, Ge M, Wickert J, Schuh H (2016) Tropospheric delay parameters from numerical weather models for multiGNSS precise positioning. Atmos Meas Tech 9:5965-5973. https://doi. org/10.5194/amt-9-5965-2016

Lu C, Li X, Zus F, Heinkelmann R, Dick G, Ge M, Schuh H (2017) Improving BeiDou real-time precise point positioning with numerical weather models. J Geod 91(9):1019-1029. https://doi.org/10.1007/s00190-017-1005-2

Montenbruck O et al (2017) The Multi-GNSS Experiment (MGEX) of the International GNSS Service (IGS) - achievements, prospects and challenges. Adv Space Res 59(7):1671-1697

Niell AE (1996) Global mapping functions for the atmosphere delay at radio wavelengths. J Geophys Res 101(B2):3227-3246

Reigber C, Gendt G, Dick G, Tomassini M (2001) Near real-time water vapor monitoring in a German GPS network and assimilation into weather forecast model. In: 14th international technical meeting of the Satellite Division of the Institute of Navigation (ION GPS 2001), Salt Lake City, UT, pp 1084-1093

Saastamoinen J (1972) Contributions to the theory of atmospheric refraction. Bull Géod 105(1):279-298

Shi J, Xu C, Guo J, Gao Y (2014) Local troposphere augmentation for realtime precise point positioning. Earth Planets Space 66:30. https://doi. org/10.1186/1880-5981-66-30 
Uhlemann M, Gendt G, Ramatschi M, Deng Z (2015) GFZ global multi-GNSS network and data processing results. In: IAG 150 years. Springer, Berlin, pp 673-679

Zhang X, Zhu F, Li P, Zhai G (2013) Zenith troposphere delay interpolation model for regional cors network augmented PPP. Geomat Inf Sci Wuhan Univ 38(6):679-683. https://doi.org/10.13203/j.whugis2013.06.022 (In Chinese)
Zumberge JF, Heflin MB, Jefferson DC, Watkins MM, Webb FH (1997) Precise point positioning for the efficient and robust analysis of GPS data from large networks. J Geophys Res 102(B3):5005-5017

Zus F, Dick G, Douša J, Heise S, Wickert J (2014) The rapid and precisecomputation of GPS slant total delays and mapping factors utilizing a numericalweather model. Radio Sci 49(3):207-216

\section{Submit your manuscript to a SpringerOpen ${ }^{\circ}$ journal and benefit from:}

- Convenient online submission

- Rigorous peer review

- Open access: articles freely available online

- High visibility within the field

- Retaining the copyright to your article

Submit your next manuscript at $>$ springeropen.com 